\title{
Tyrosine kinase receptors as molecular targets in pheochromocytomas and paragangliomas
}

Clarissa A Cassol ${ }^{1}$, Daniel Winer ${ }^{1}$, Wei Liu ${ }^{2}$, Miao Guo ${ }^{2}$, Shereen Ezzat ${ }^{2,3}$ and Sylvia L Asa ${ }^{1,2}$

${ }^{1}$ Department of Pathology, University Health Network, Toronto, ON, Canada; ${ }^{2}$ Ontario Cancer Institute, University Health Network, Toronto, ON, Canada and ${ }^{3}$ Department of Medicine, University Health Network, Toronto, ON, Canada

\begin{abstract}
Pheochromocytomas and paragangliomas are neuroendocrine tumors shown to be responsive to multitargeted tyrosine kinase inhibitor (TKI) treatment. Despite growing knowledge regarding their genetic basis, the ability to predict behavior in these tumors remains challenging. There is also limited knowledge of their tyrosine kinase receptor expression and whether the clinical response observed to the TKI sunitinib relates only to its antiangiogenic properties or also due to a direct effect on tumor cells. To answer these questions, an in vitro model of sunitinib treatment of a pheochromocytoma cell line was created. Sunitinib targets (VEGFRs, PDGFRs, and C-KIT), FGFRs, and cell cycle regulatory proteins were investigated in human tissue microarrays. SDHB immunohistochemistry was used as a surrogate marker for the presence of succinate dehydrogenase mutations. The FGFR4 G388R single nucleotide polymorphism was also investigated. Sunitinib treatment in vitro decreases cell proliferation mainly by targeting cell cycle, DNA metabolism, and cell organization genes. FGFR1, -2, and -4, VEGFR2, PDGFR $\alpha$, and p16 were overexpressed in primary human pheochromocytomas and paragangliomas. Discordant results were observed for VEGFR1, p27, and p21 overexpressed in paragangliomas but underexpressed in pheochromoctyomas; PDGFR/, Rb, and Cyclin D1 overexpressed in paragangliomas only; and FGFR3 overexpressed in pheochromocytomas and underexpressed in paragangliomas. Low expression of C-KIT, p53, and Aurora kinase A and B was observed. Nuclear FGFR2 expression was associated with increased risk of metastasis (odds ratio $(O R)=7.61, P=0.008)$, as was membranous PDGFR $\alpha$ (OR $=13.71$, $P=0.015)$, membranous VEGFR1 (OR $=8.01, P=0.037$ ), nuclear MIB1 $(O R=1.26, P=0.008)$, and cytoplasmic p27 (OR=1.037, $P=0.030)$. FGFR3, VEGFR2, and C-KIT levels were associated with decreased risk of metastasis. We provide new insights into the mechanistic actions of sunitinib in pheochromoctyomas and paragangliomas, and support current evidence that multitargeted TKIs might be a suitable treatment alternative for these tumors.
\end{abstract}

Modern Pathology (2014) 27, 1050-1062; doi:10.1038/modpathol.2013.233; published online 3 January 2014

Keywords: paragangliomas; pheochromoctyomas; sunitinib; tyrosine kinase receptors

Pheochromocytomas and paragangliomas are tumors of neural crest-derived endocrine cells throughout the distribution of the sympathetic and parasympathetic nervous system. ${ }^{1}$ The discovery of succinate dehydrogenase $(S D H)$ mutations as a frequent underlying cause of paragangliomas in the year 2000 has launched a phase of accelerated gene discovery in these tumors; it is now known that a genetic predisposition is present in almost $30 \%$ of

Correspondence: Dr SL Asa, MD, PhD, Department of Pathology, University Health Network, 200 Elizabeth Street, 11th Floor, Toronto, ON M5G 2C4, Canada.

E-mail: sylvia.asa@uhn.ca

Received 3 September 2013; revised 15 October 2013; accepted 16 October 2013; published online 3 January 2014 cases. ${ }^{2}$ However, despite growing knowledge of their genetics, the ability to predict behavior in these tumors remains challenging. Numerous factors have been associated with malignancy, including the presence of $S D H B$ mutations, ${ }^{2}$ high proliferative index, ${ }^{3,4}$ and tumor size and location; ${ }^{5}$ however, the only accepted criterion of malignancy is the presence of distant metastasis. ${ }^{1}$

When malignant, pheochromocytomas and paragangliomas do not usually respond to traditional therapies. Recent reports of successful treatment of malignant pheochromocytoma/paraganglioma with the multitargeted tyrosine kinase inhibitor (TKI) sunitinib provided clinical evidence that receptor tyrosine kinases (RTKs) might be involved in the development or progression of these tumors., 
RTKs and their ligands are known to be mutated or overexpressed in a variety of endocrine malignancies, including thyroid, ${ }^{8,9}$ pituitary, ${ }^{10}$ pancreas, ${ }^{11}$ pheochromocytomas, ${ }^{3,12-15}$ and paragangliomas. ${ }^{16}$ Single nucleotide polymorphisms (SNPs) in RTK genes may also have a role in the development or progression of tumors, as is the case with the common FGFR4 G388R SNP. ${ }^{17}$

As therapeutic response to TKIs in tumor models has been shown to be not only due to an antiangiogenic effect but also due to a direct effect on tumor cells, ${ }^{18,19}$ we decided to investigate this possibility by creating an in vitro model of sunitinib treatment using a mouse pheochromocytoma cell line (MPC 4/30). ${ }^{20}$ In addition, tissue microarrays from human tumors were constructed and stained with antibodies against the main sunitinib potential targets (VEGFRs, PDGFRs, and C-KIT), as well as other RTKs (FGFRs) that might be related to the development of therapeutic resistance. ${ }^{11,21}$ Following our initial observations that in vitro treatment of pheochromocytoma cells results in significantly altered expression of cell cycle regulatory proteins, we further expanded the tissue microarray immunohistochemistry panel to include cell cycle regulatory proteins (Cyclin D1, Rb, p16, p21, p27, p53, MIB1, and Aurora A and B). SDHB immunohistochemistry was used as a surrogate marker for $S D H$ mutations, ${ }^{22}$ and genotyping for the common FGFR4 G388R polymorphism was performed in order to assess a possible influence of this SNP on the development and progression of these tumors. ${ }^{17,23}$

\section{Materials and methods}

\section{In vitro Studies: Sunitinib Treatment of a Mouse Pheochromocytoma Cell Line}

The MPC 4/30 mouse pheochromocytoma cell line was kindly provided by Dr A Tischler (Tufts Medical Center, Boston, MA, USA) and maintained as previously described. ${ }^{20}$ Sunitinib malate $100 \mathrm{mg}$ was purchased from Sequoia Research Products (Pangbourne, UK; SRP01785s) and dissolved in dimethyl sulfoxide to obtain a $25-\mathrm{mM}$ solution. Cells were cultured on $10-\mathrm{cm}$ plates. After trypsinization, two million cells were plated, grown in supplemented medium for $24 \mathrm{~h}$, starved in serum-free medium for $24 \mathrm{~h}$, then treated with sunitinib malate in different concentrations $(0,2.5$, and $5.0 \mu \mathrm{M}$ ) for $72 \mathrm{~h}$. Cells were then trypsinized and divided for flow cytometry and RNA expression analysis. For flow cytometry, $1-3 \times 10^{6}$ cells were washed with PBS and fixed with cold $80 \%$ ethanol for $1 \mathrm{~h}$ on ice. Fixed cells were washed with staining buffer $(0.2 \%$ Triton X-100 and $1 \mathrm{mmol} / \mathrm{l}$ EDTA, $\mathrm{pH}$ 8.0, in PBS) and resuspended in the staining buffer containing $50 \mu \mathrm{g} / \mathrm{ml}$ RNase A (Sigma-Aldrich, St Louis, $\mathrm{MO}, \mathrm{USA}$ ) and $50 \mu \mathrm{g} / \mathrm{ml}$ propidium iodide for $1 \mathrm{~h}$.
Cell cycle analysis was performed by FACS Caliber (BD Biosciences, Franklin Lakes, NJ, USA) using Cellquest analysis and specific $S$ phase was analyzed using Modfit DNA Analysis (Verity Software House, Topsham, ME, USA). For microarray analysis, RNA was extracted using RNAeasy (Qiagen, Hilden, Germany) and analyzed using Gene 1.0 ST Array (Affimetrix, Santa Clara, CA, USA). Results were examined using DAVID Bioinformatic Resources 6.7 (Frederick, MD, USA) and String protein association network software V9.0. ${ }^{24}$

\section{Human Tumor Studies}

Case selection, tissue microarray construction and image analyses. Following institutional ethics board approval, 153 cases of pheochromocytomas/ paragangliomas were identified in our institutional files from 2001 to 2009. Of these, 132 tumors (82 paragangliomas, 43 pheochromocytomas, and 7 metastases) from 115 patients provided enough tissue for tissue microarray construction. Triplicate or quadruplicate cores of tumor and normal adrenal medulla (when available) were included in the tissue microarray. Relevant clinical and histopathological data were recorded. Tissue microarrays were stained with antibodies against FGFR1 (Abcam, Cambridge, MA, USA), FGFR2 (SC-122, Santa Cruz Biotechnology, Santa Cruz, CA, USA), FGFR3 (SC123, Santa Cruz Biotechnology), FGFR4 (SC-124, Santa Cruz Biotechnology), C-KIT (A4502, Dako, Carpinteria, CA, USA), PDGFR $\alpha$ (Abcam, ab-61219), PDGFR $\beta$ (sc-432, Santa Cruz Biotechnology), VEGFR1 (1301-1, Epitomics, Burlingame, CA, USA), VEGFR2 (2479, Cell Signaling, Beverly, MA, USA), SDHB (HPA002868, Sigma-Aldrich Corp), MIB1 (Novus Biologicals, Littleton, CO, USA, NB-110-90592), p16 (CINtect Histology Kit, Heidelberg, Germany), p21 (Pharmingen, San Diego, CA, USA, 556431), p27 KIP1 (BD Transduction Laboratories, 610242), p53 (Novocastra, Newcastle, UK), Ki67 (Novus Biologicals), Cyclin D1 (Lab Vision, Fremont, CA, USA), Rb (Pharmingen), and SDHB (Sigma-Aldrich Corp). Immunohistochemistry stained slides were scanned using ScanScope (Aperio, Vista, CA, USA) and analyzed using Spectrum Plus (Aperio) with algorithms to determine nuclear, membrane, cytoplasmic, and overall immunostaining. Outputs generated included the percentage of positive nuclei and nuclear intensity score ranging from 0 to 3 , according to the average intensity of positive nuclei; the percentage of cells with positive membrane staining and a membrane final score ranging from 0 to 3 , according to the percentages of strong, moderate, and weak staining cells; and an overall staining score calculated by giving different weights to the percentage areas of weak, medium, and strong immunostaining $(1 *(\%$ Weak $)+2 *(\%$ Medium $)+$ $3 *(\%$ Strong $))$. Unless otherwise specified, the use of the term 'score' throughout the text refers to the 
overall staining score. All algorithms were optimized to ensure that the outputs generated corresponded to the pathologist interpretation of the immunostaining intensity and percentage.

Tumors with either completely negative staining or weak positivity for SDHB by immunohistochemistry were considered SDHB-deficient tumors. These are referred to as $S D H$ related, in accordance with the fact that they can be due to either mutations or epigenetic changes in $S D H A, S D H B, S D H C$, or $S D H D$, or in genes involved in mitochondrial respiratory complex II assembly or regulation. ${ }^{22}$

Immunohistochemistry heat maps and hierarchical clustering. The heat maps were generated using Multiple Experiment Viewer software version 4.8.1.25 The parameter settings for hierarchical clustering were based on the Pearson's correlation distance metric and the average linkage method. Rows represent the type of tumor and its genetic background. Columns represent the immunohistochemical stains. Green indicates the lowest expression, black indicates intermediate expression, and red indicates the highest expression of the overall immunohistochemistry score given for each tumor sample. The color scale bar is shown at the top of the heat maps.

FGFR4 genotyping. DNA was extracted using the phenol-chlorophorm method from paraffin cores collected at the time of tissue microarray construction. Whenever available, preference was given to normal tissue DNA (adjacent adrenal, lymph nodes, or tissue available from other samples from the same patient). Exon 9 of $f g f r 4$ was PCR amplified and RFLP digested with BstN1 to distinguish three FGFR4 genotypes: wild type (Gly/Gly), heterozygous $\mathrm{Gly}^{388}$ (Gly/Arg), and homozygous $\mathrm{Arg}^{388}$ (Arg/Arg) as previously described. ${ }^{23}$

\section{Statistical Analyses}

Continuous variables are expressed as mean \pm s.d. Categorical variables are expressed as the absolute number and percentages. Comparisons between two continuous variables were performed using the Mann-Whitney test or the $t$-test. For comparisons between categorical variables, the $\chi^{2}$-test or the Fischer's exact test were used, according to the distribution of the variable. Correlations between continuous variables were tested using the Pearson's correlation coefficient. Univariate logistic regression analysis was used to determine whether clinical characteristics, FGFR4 genotypes, and levels of expression of RTKs were predictors of the outcomes-death, metastasis, or local recurrence. Odds ratios (ORs) and 95\% confidence intervals (CIs) were estimated as measures of the magnitude of the associations. All analyses were carried out considering pheochromocytoma cases and paraganglioma cases separately. A $P$-value $<0.05$ was considered significant. Statistical analyses were performed using the SPSS 16.0 software (Chicago, IL, USA).

\section{Results}

\section{Cell Line Treatment Analyses}

Cell cycle analysis showed that sunitinib decreases MPC 4/30 proliferation and increases apoptosis (Figure 1a). Gene expression profiling revealed that sunitinib strongly downregulates cell cycle-associated genes. DAVID functional annotation tool analysis revealed that in tumor cells treated with sunitinib, $\sim 37 \%$ of all genes (125/339) with $>2$-fold downregulation, are linked to the cell cycle (Supplementary Table 1), of which $>50 \%$ affect the $M$ phase (summarized in Figure $1 \mathrm{~b}$ as a gene interaction network). Of genes upregulated $>2 \times$ by sunitinib, stress response proteins were some of the most prominently inolved (12/63 proteins or $19 \%)$, (Supplementary Table 2). A summary of the biological processes affected by sunitinib treatment in vitro is depicted in Figure 1c.

\section{Clinical and Histopathological Data, Outcomes, and SDH Status}

Of 115 patients, 39 had pheochromocytomas and 76 had paragangliomas. According to the clinical notes, 8 patients had MEN2, 2 patients had Neurofibromatosis type 1, and 2 patients had von Hippel-Lindau Syndrome; 46 cases showed SDHB loss by immunohistochemistry. Their main clinical and histopathological characteristics and FGFR4 genotypes are summarized in Table 1. Additional tumor characteristics (location, size, and weight) are described in Supplementary Table 3.

No association was found between age, gender, or family history and the outcomes. Median follow-up was 23 months. Of the nine malignant cases, six were SDHB deficient. SDHB-intact and -deficient tumors differed significantly in several of the parameters analysed (Figure 2).

\section{Sunitinib Targets Expression in Pheochromocytomas and Paragangliomas, and Clinical Associations}

Differential expression of the sunitinib targets VEGFR1 and 2, PDGFR $\alpha$ and $\beta$ in pheochromocytomas, paragangliomas, and normal medulla is depicted in Figures 3 and 4, and Supplementary Figures 1-3. Hierarchical clustering of tumors based on an immunohistochemistry heat map showed distinct segregation of pheochromocytomas from paragangliomas. $S D H$-related tumors tend to cluster together, as well as MEN2-associated tumors, which tended to have lower levels of VEGFRs. 

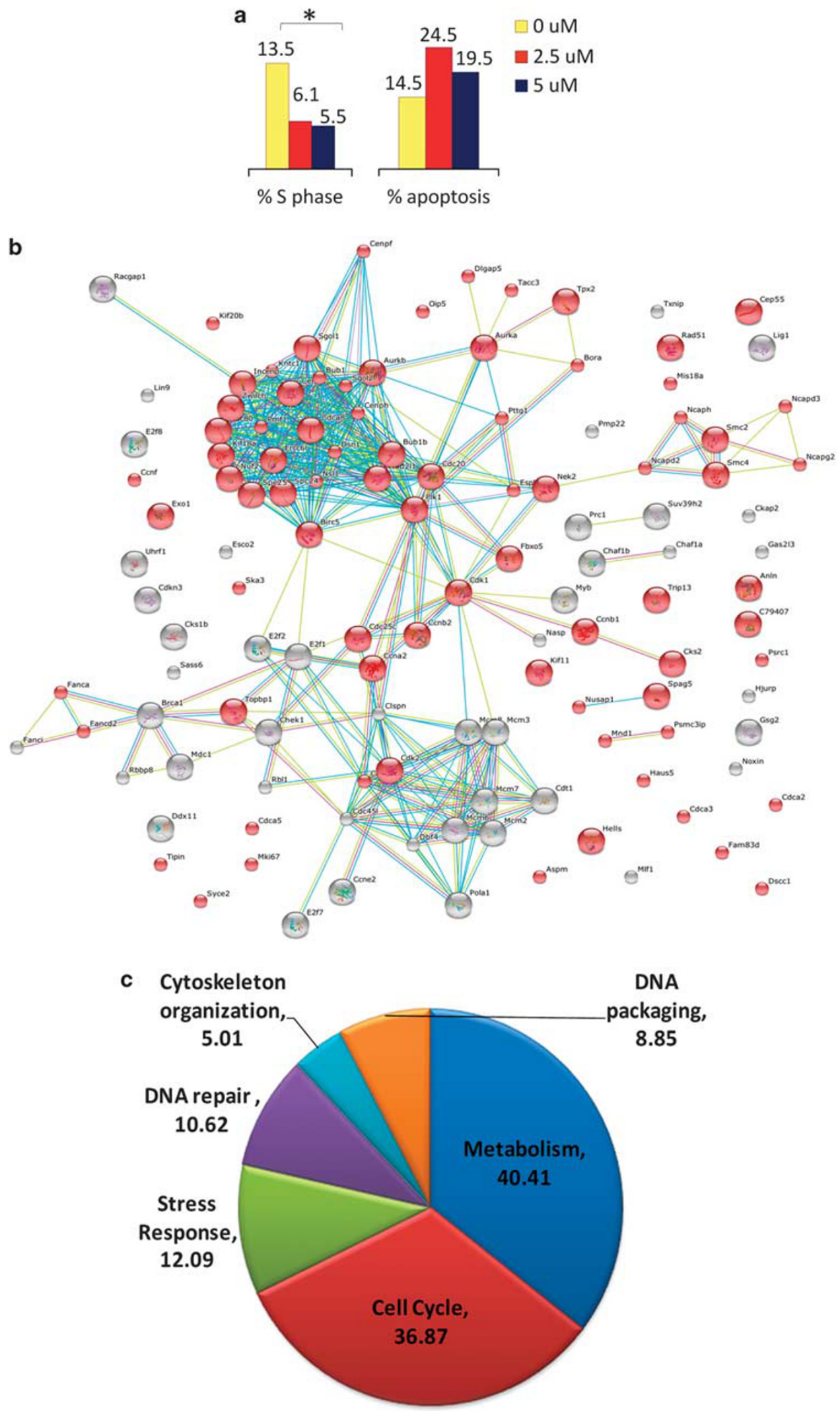

Figure 1 Sunitinib treatment of the mouse pheochromocytoma MPC 4/30 cell line in vitro. (a) Cell cycle analyses of MPC 4/30 cells treated with 2.5 and $5 \mu \mathrm{M}$ of sunitinib show a sigificant inhibition of replication compared with controls receiving $0 \mu \mathrm{M}\left({ }^{*} P<0.05\right)$. Values shown represent means derived from three different experiments. (b) More than $50 \%$ of cell cycle proteins $>2 \times$ downregulated by sunitinib affect the M phase of mitosis. (c) The major biological processes affected by sunitinib in MPC 4.30 cells are demonstrated. 
Table 1 Clinical characteristics of patients

\begin{tabular}{|c|c|c|c|c|}
\hline Variable & $\begin{array}{c}\text { All } \\
\text { patients } \\
(\mathrm{n}=115)\end{array}$ & $\begin{array}{c}\text { Pheo } \\
(\mathrm{n}=39)\end{array}$ & $\begin{array}{c}\text { Para } \\
(\mathrm{n}=76)\end{array}$ & $\mathrm{P}$-value \\
\hline $\begin{array}{l}\text { Age at diagnosis } \\
\text { (years) }\end{array}$ & $46.8 \pm 12.3$ & $45.9 \pm 13.0$ & $47.3 \pm 12.0$ & 0.571 \\
\hline Female gender & $71(61.7)$ & $22(56.4)$ & $49(64.5)$ & 0.424 \\
\hline \multicolumn{5}{|l|}{$\begin{array}{l}\text { FGFR4 genotype } \\
(\mathrm{n}=105)\end{array}$} \\
\hline $\begin{array}{l}\text { G388-G388 } \\
\text { (Gly/Gly) }\end{array}$ & $38(33)$ & $9(23.1)$ & $29(38.2)$ & 0.64 \\
\hline $\begin{array}{l}\text { G388-R388 } \\
\text { (Gly/Arg) }\end{array}$ & $68(59)$ & 30 (76.9) & $38(50)$ & \\
\hline Unknown & $9(7.8)$ & $0(-)$ & $9(11.8)$ & \\
\hline SDHB loss by IHC & $46(40)$ & $1(2.6)$ & $45(59)$ & $<0.001$ \\
\hline Bilateral/multiple & $20(17.4)$ & $4(10.3)$ & $17(22.4)$ & 0.132 \\
\hline Family history & $10(8.6)$ & $4(10.3)$ & $5(6.6)$ & 0.486 \\
\hline Metastatic disease & $9(7.8)$ & $0(-)$ & 9 (11.8) & 0.093 \\
\hline Local recurrence & $4(3.5)$ & $0(-)$ & $4(5.3)$ & 0.298 \\
\hline $\begin{array}{l}\text { Death related to } \\
\text { tumour }\end{array}$ & $2(1.7)$ & $0(-)$ & $2(2.6)$ & 0.548 \\
\hline
\end{tabular}

Abbreviations: N, number; Para, paragangliomas; Pheos, pheochromocytomas.

Values are means \pm s.d. Numbers in brackets are percentage of an absolute number.

C-KIT expression was low in both tumor types and did not differ significantly from normal medulla (Supplementary Figure 1).

VEGFR1 score was higher in paragangliomas, in males (128.3 vs $111.1 \pm 42.8, P=0.025)$ and $S D H$ related tumors (Figures 2 and 4), but lower in pheochromocytomas (Figures 3 and 4). Overall staining and membrane scores were higher in tumors that metastasized (152.3 vs $111, P=0.01$ and 1.8 vs $1.3 \pm, P=0.022$, respectively) and were associated with increased risk of metastases $(\mathrm{OR}=1.05 ; \quad 95 \% \quad \mathrm{CI}=1.01-1.10 ; \quad P=0.021$ for VEGFR1 score and OR $=8.01 ; 95 \% \mathrm{CI}=1.13-56.80$; $P=0.037$ for VEGFR1 membrane score).

VEGFR2 was overexpressed in both tumor types (Figures 3 and 4), but tumors that metastasized had lower scores $(67.4$ vs 90.0, $P=0.011)$. It was associated with a decreased risk of metastasis $(\mathrm{OR}=0.97,95 \% \mathrm{CI}=0.94-0.99, P=0.017)$.

PDGFR $\alpha$ score was higher in both tumor types (Figures 3 and 4 ) and in tumors that metastasized (73.5 vs 53.2, $P=0.005$ ). PDGFR $\alpha$ membrane score was higher in tumors that metastasized (1.6 vs 1.3, $P=0.008)$ or died of the disease $(2.0$ vs 1.2 , $P=0.003$ ). It was associated with a significantly increased risk for metastasis $(\mathrm{OR}=13.71,95 \%$ $\mathrm{CI}=1.65-113.81, P=0.015)$.

PDGFR $\beta$ score was higher in paragangliomas (Figures 3 and 4), $S D H$-related tumors (Figures 2 and 4), and tumors that metastasized (23.0 vs 13.0, $P=0.032$ ). It was also higher in tumors from patients with a positive family history of pheochromocytoma/paraganglioma (22.5 vs $12.9, P=0.0031)$ and patients with FGFR4 Gly/Gly genotype (17.2 vs 11.7 in Gly/Arg patients, $P=0.045$ ), and was associated with an increased risk of metastasis $(\mathrm{OR}=1.04,95 \%$ $\mathrm{CI}=1.01-1.09, P=0.043$ ).

C-KIT levels were significantly lower in $\mathrm{SDH}$ related tumors (Figure 2) and those that metastasized (13.7 vs 24.1, $P=0.033$ ).

\section{FGFR1, 2, 3, and 4 Expression in Pheochromocytomas} and Paragangliomas, and Clinical Associations

FGFR1, 2, and 4 scores were higher in pheochromocytomas and paragangliomas compared with that in normal medulla (Figure 3). FGFR1, 2, and 4 expression was also higher in $S D H$-related tumors (Figure 2). In contrast, FGFR3 was overexpressed in pheochromocytomas but underexpressed in paragangliomas (Figures 3 and 4), and showed lower levels in $S D H$-related tumors (Figure 2). Additional data, including membrane and nuclear differential expression of FGFRs in pheochromocytomas and paragangliomas, can be found in Supplementary Figure 4.

FGFR1 score was higher in tumors that metastasized (117.5 vs $94 ; P=0.01$ ) and was associated with increased risk for metastases $(\mathrm{OR}=1.03,95 \%$ $\mathrm{CI}=1.01-1.05, P=0.027)$. FGFR2 nuclear score was higher in tumors that metastaszed (1.7 vs 1.3; $P=0.002$ ) and was associated with increased risk of metastasis $(\mathrm{OR}=7.61,95 \% \quad \mathrm{CI}=1.70-34.17$, $P=0.008$ ). Conversely, FGFR3 score was significantly lower in tumors that metastasized (31.3 vs 49.0; $P=0.006$ ). In paragangliomas, an inverse correlation was found between the tumor size and the percentage and intensity of FGFR3 membrane staining $(r=-0.364, P=0.001$ and $r=-0.277, P=0.022)$. FGFR4 score was higher in tumors from patients with a positive family history of pheochromocytoma/paraganglioma $(24.6$ vs $13.7, \quad P=0.002)$. A positive correlation between tumor size and FGFR4 percentage and intensity of membrane staining was present in paragangliomas $(r=0.240, P=0.38$ and $r=0.260, P=0.024$, respectively), whereas a positive correlation between the FGFR4 overall staining score and MIB1 percent-positive nuclei was observed in pheochromocytomas $(r=0.359, P=0.025)$.

\section{FGFR4 Genotyping of Pheochromocytomas and Paragangliomas, and Clinical Associations}

From 115 patients, DNA was extracted from either normal $(N=107)$ or tumor $(N=8)$ tissue. In nine cases, genotyping could not be performed. There was no significant difference in the distribution of these alleles between pheochromocytomas and paragangliomas (Table 1). No association was found between FGFR4 genotype and FGFR4 levels of expression, nor with age, gender, bilateral/multiple tumors, family history, any individual outcomes, or the combined outcome.

A comparison between the distribution of FGFR4 alleles in our cases and those of normal populations 

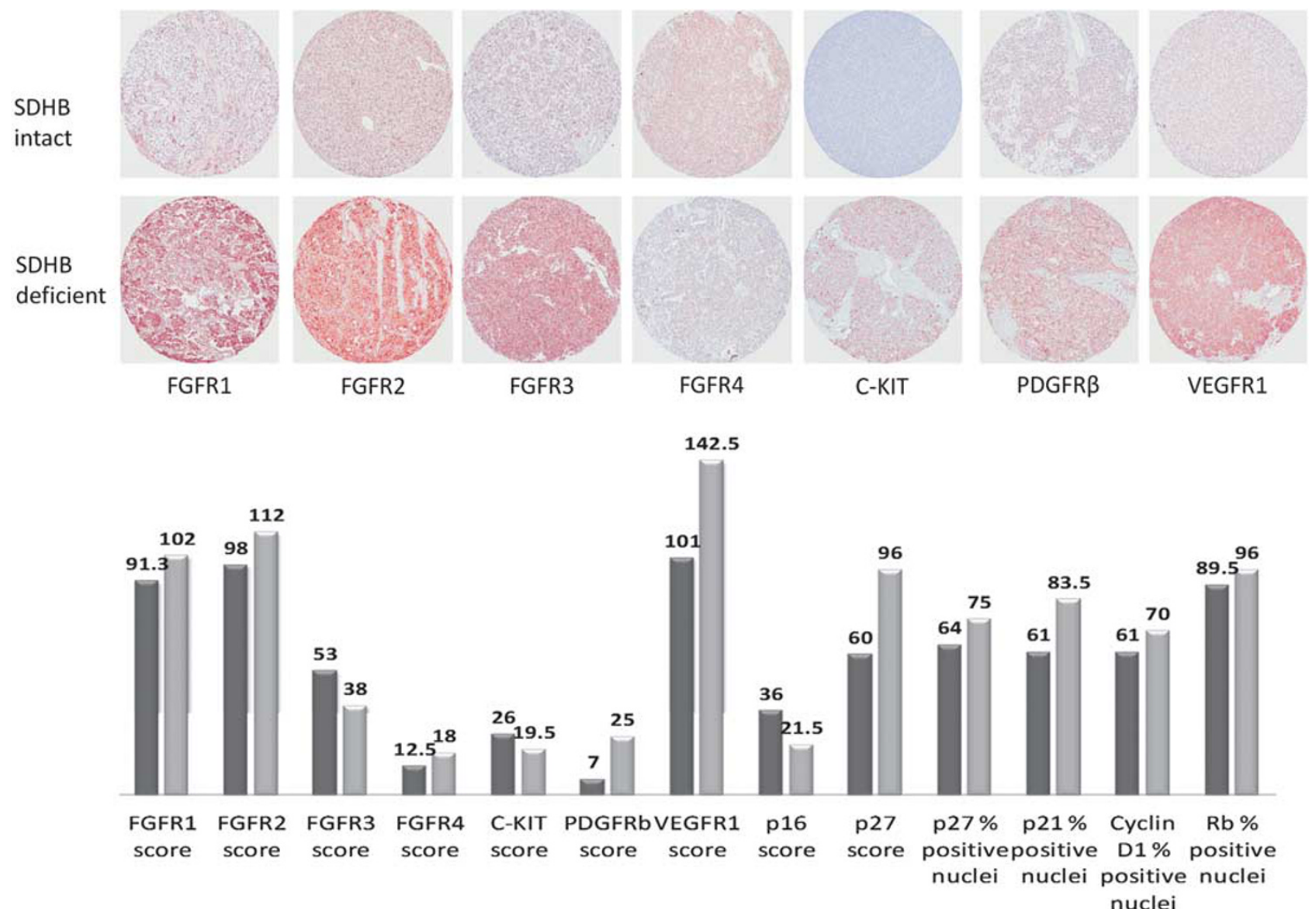

国 SDHB intact $\square$ SDHB deficient

nuclei

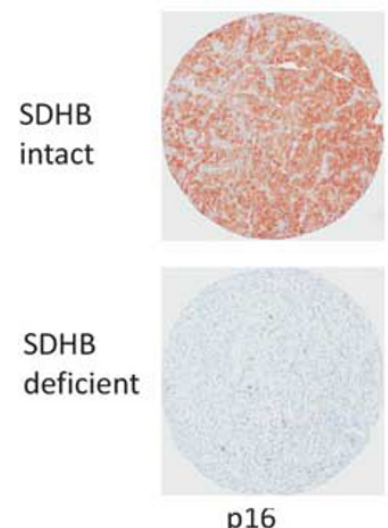

p16
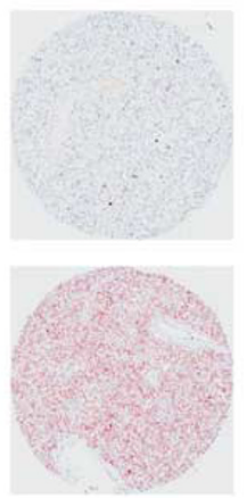

p21
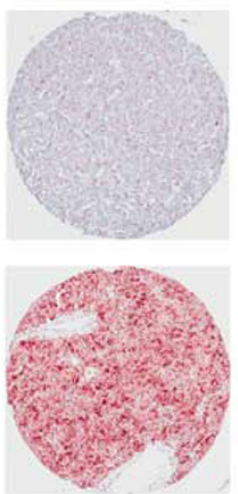

p27
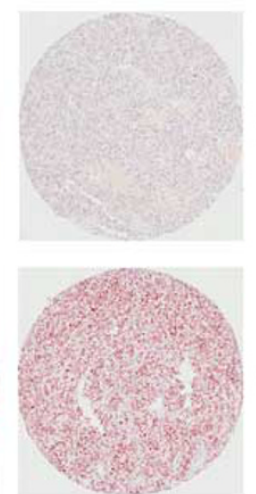

$\mathrm{Rb}$
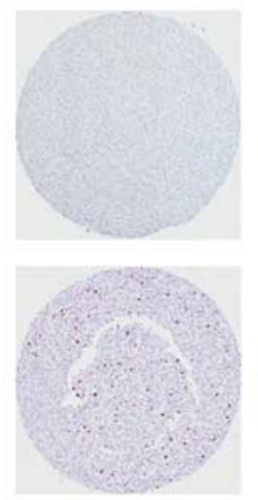

MIB1
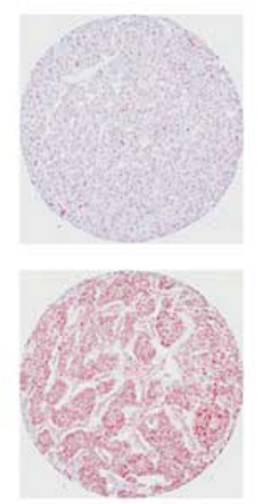

Cyclin D1

Figure 2 Differential expression of receptor tyrosine kinases (RTKs) and cell cycle markers between SDHB-intact and SDHB-deficient tumors. SDHB-deficient tumors $(n=46)$ are considered to be succinate dehydrogenase $(S D H)$-related. Only significant results $(P<0.05)$ are shown, along with representative tissue microarray spots. Score refers to the overall staining score (values range: $0-300$ ).

previously reported in the literature is depicted in Table 2.

\section{Cell Cycle Markers in Pheochromocytomas and Paragangliomas, and Clinical Associations}

The differential expression of cell cycle markers in pheochromocytomas, paragangliomas, and normal medulla is depicted in Figure 5, with selected markers also shown in Figure 4 and additional outputs analyzed in Supplementary Figure 5. Aurora B expression was negligible (not shown). Cell cycle marker expression according to the SDHB immunohistochemistry status of tumors is illustrated in Figure 2.

Tumors that metastasized had a higher percentage of cytoplasmic p27 staining $(53.5 \%$ vs $39.1 \%$, $P=0.045)$ and MIB1 percent-positive nuclei $(5.8 \%$ 

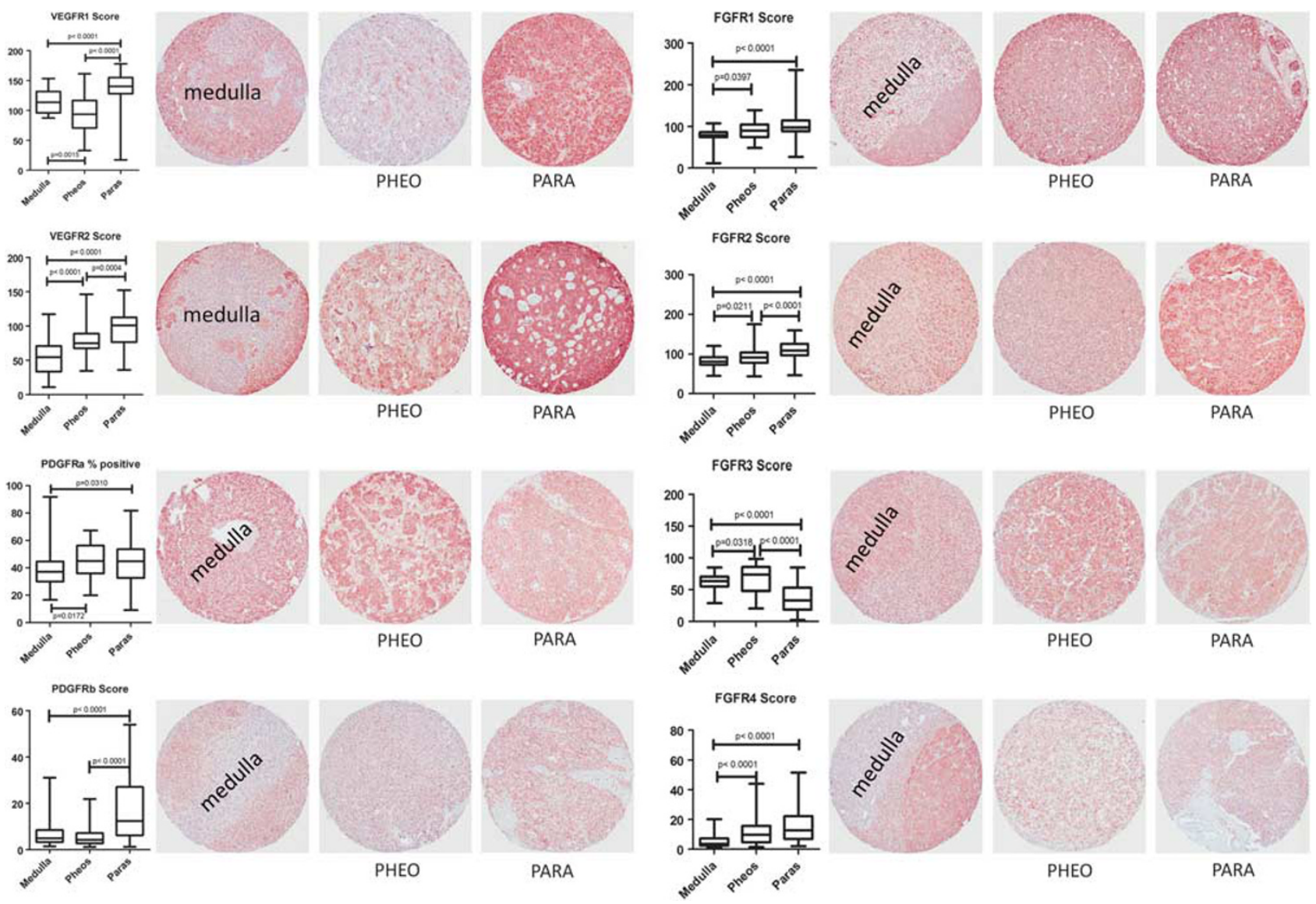

Figure 3 Expression of VEGFRs, PDGFRs, and FGFRs in pheochromocytomas, paragangliomas, and normal adrenal medulla. Representative tissue microarray spots are shown and staining score values ranging from 0 to 300 are graphed. Pheos, pheochromocytomas; paras, paragangliomas.

vs $2.5 \%, P=0.002)$. MIB1 labeling index (LI) was associated with increased risk of metastasis $(\mathrm{OR}=1.26,95 \% \mathrm{CI}=1.06-1.49, P=0.008)$.

Tumors from patients with a family history of pheochromocytoma/paraganglioma had a higher p16 score (50.75 vs 28.1, $P=0.033$ ) and a higher percentage of p16 cytoplasmic staining $(30.7 \%$ vs $11.5 \%, P=0.002$ ). Bilateral or multiple tumors had a higher percentage of Rb-positive nuclei $(95.6 \%$ vs $91.2 \% ; P=0.033$ ).

Tumors from patients carrying an FGFR4-R388 allele had a higher percentage of nuclei positive for p53 $(2.1 \%$ vs $1 \%, P=0.014)$ and a lower percentage for Cyclin D1 (60.9\% vs 70.6\%, $P=0.002)$.

\section{Discussion}

\section{Sunitinib Potential Targets in Pheochromocytomas and Paragangliomas}

We have demonstrated that sunitinib treatment decreases cell proliferation in mouse pheochromocytoma cells in vitro mainly by affecting cell cycle, DNA metabolism, and cell organization genes. It also increases apoptosis, which is in keeping with similar observations of sunitinib treatment of a rat pheochromocytoma cell line. ${ }^{19}$

Among sunitinib potential targets, VEGFR2 and PDGFR $\alpha$ were overexpressed in pheochromocytomas and paragangliomas, whereas PDGFR $\beta$ was overexpressed in paragangliomas only and VEGFR1 was overexpressed in paragangliomas but underexpressed in pheochromocytomas. Membranous PDGFR $\alpha$ and VEGFR1 were associated with an increased risk of metastatic disease. Our finding of high VEGFR levels in these tumors is consistent with previous observations. ${ }^{12-14}$ In addition, VEGF ${ }^{13,14}$ and VEGFR1 (ref. 14) overexpression might be predictors of malignant behavior. Given that VEGF/ VEGFR expression are regulated by HIF $1 \alpha$ and HIF $2 \alpha$, it is not surprising that we found higher levels of VEGFR1 in SDHB-deficient tumors, which is also in keeping with previous studies showing a high expression of angiogenic markers in $S D H$ - and VHL-related tumors. ${ }^{14}$ VEGFR1 expression has also been associated with poor prognostic features in thyroid $^{26}$ and hepatocellular carcinoma. ${ }^{27}$

The role of PDGFRs and their ligands in human malignancies is well documented. ${ }^{28}$ In papillary 


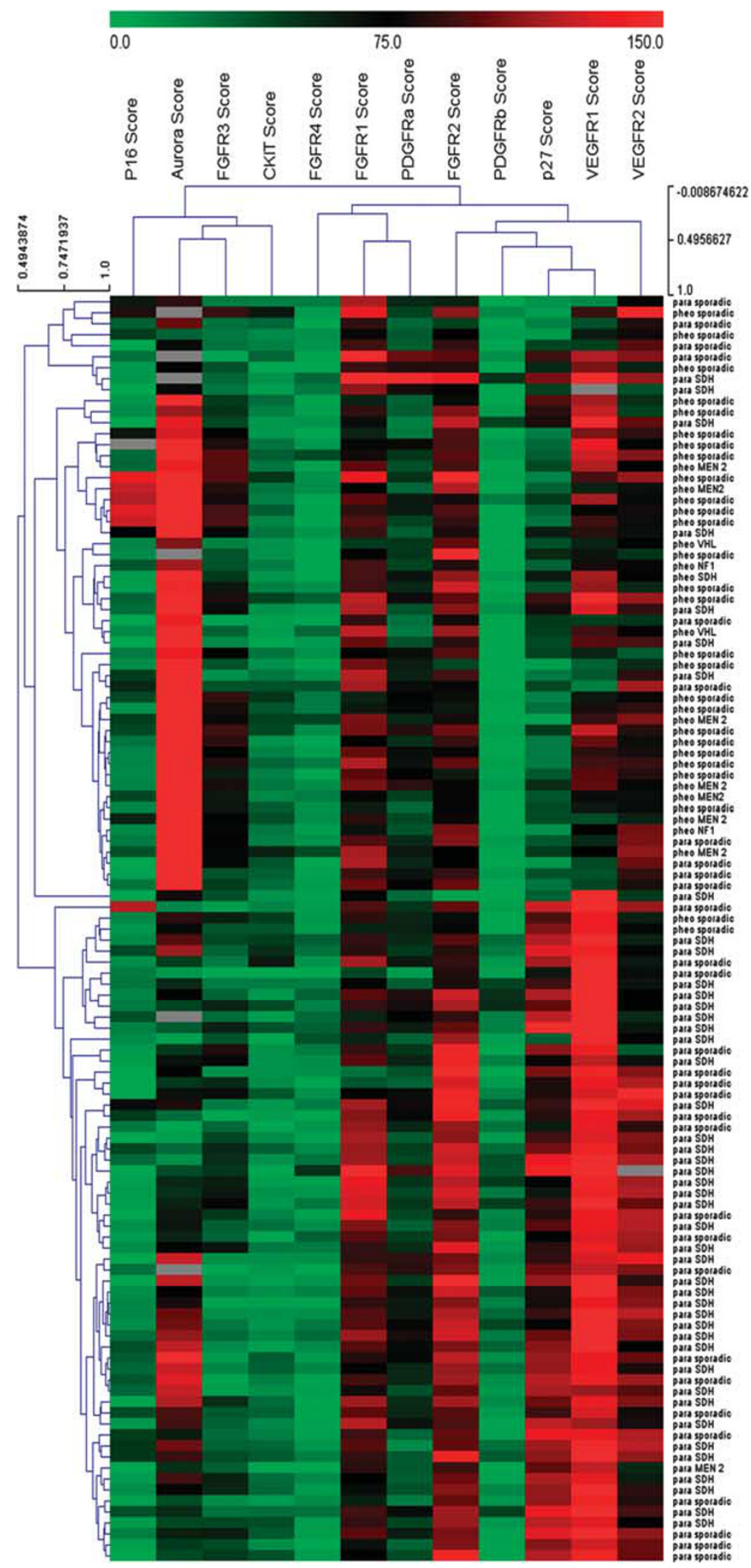

Figure 4 Heat map summary of immunohistochemical expression of selected markers in paragangliomas and pheochromocytomas. Rows represent the type of tumor and its genetic background. Columns represent the immunohistochemical stains. Green indicates the lowest expression, black indicates intermediate expression, and red indicates the highest expression of the overall immunohistochemistry score given for each tumor sample. Pheo, pheochromocytoma; para, paraganglioma. 
Table 2 Distribution of FGFR4 codon 388 SNP alleles in normal populations (18) and in patients with pheo and paras from our series

\begin{tabular}{|c|c|c|c|c|c|c|}
\hline & Gly/Gly (\%) & $\mathrm{P}$-value & Gly/Arg (\%) & $\mathrm{P}$-value & $\operatorname{Arg} / \operatorname{Arg}(\%)$ & $\mathrm{P}$-value \\
\hline Present study & 33 & & 59 & & 0 & \\
\hline Bange et $a l^{23}$ & 45 & 0.0460 & 49 & 0.0015 & 6 & 0.002 \\
\hline Morimoto 2003 & 38.2 & 0.8900 & 49 & 0.0432 & 12.7 & $<0.0001$ \\
\hline Wang 2004 & 54.6 & 0.0100 & 41.2 & 0.0017 & 4.1 & 0.03 \\
\hline Spinola 2005a & 50.9 & 0.0170 & 37.3 & $<0.0001$ & 11.4 & $<\mathbf{0 . 0 0 0 1}$ \\
\hline Spinola $2005 b$ & 48.1 & 0.0400 & 41.9 & $<0.0001$ & 10 & $<\mathbf{0 . 0 0 0 1}$ \\
\hline Yang 2008 & 32 & 0.3200 & 50.6 & 0.0176 & 17.4 & $<0.0001$ \\
\hline Ма 2008 & 37.4 & 1.0000 & 48.6 & 0.0188 & 14 & $<0.0001$ \\
\hline Han 2009 & 34.1 & 0.6000 & 43.2 & 0.0052 & 22.7 & $<0.0001$ \\
\hline FitzGerald 2009 & 50.4 & 0.0050 & 39.6 & $<\mathbf{0 . 0 0 0 1}$ & 9.9 & $<0.0001$ \\
\hline Naidu 2009 & 52.4 & 0.0060 & 41.6 & $<\mathbf{0 . 0 0 0 1}$ & 6 & 0.0002 \\
\hline Tanuma 2010 & 42 & 0.0420 & 48 & 0.03 & 10 & $<0.0001$ \\
\hline Ho 2010 & 51.5 & 0.0070 & 40.2 & $<0.0001$ & 8.2 & 0.003 \\
\hline
\end{tabular}

Abbreviations: Para, paragangliomas; Pheos, pheochromocytomas; SNP, single nucleotide polymorphism. Bold highlights significant values $(P<0.05)$.

The references listed in this table have been extracted from ref.18.

thyroid cancer PDGFR $\alpha$ overexpression was associated with lymph node metastasis, ${ }^{29}$ and in prostate adenocarcinomas PDGFR $\alpha$ expression was associated with bone metastasis. ${ }^{30}$ PDGF receptors, particularly $\operatorname{PDGFR} \alpha$, have been implicated in neural crest cell (NCC) migration and NCC-derived tissue development. ${ }^{31}$ PDGF expression in cultured cells is responsive to a variety of stimuli, including hypoxia. ${ }^{28}$ As at least a subset of pheochromocytomas ( $S D H$-related and $V H L$-associated tumors) exhibits a hypoxic gene signature, it is possible that an autocrine-paracrine PDGF-PDGFR loop might be in place in these tumors, as is the case with other human malignancies. ${ }^{28}$ That would be in keeping with our observation of higher PDGFR $\beta$ overall staining score in $S D H$-related tumors. Moreover, bFGF stimulates PDGFR $\alpha$ expression in smooth muscle cells in vitro. ${ }^{32}$ Overexpression of bFGF in paragangliomas ${ }^{3,15}$ could potentially contribute to PDGFR $\alpha$ overexpression and explain the positive correlation observed between expression of PDGFR $\alpha$ and the bFGF receptor FGFR1. Both are involved in the proliferation of oligodendrocyte progenitors in response to demyelination. ${ }^{33}$

Low expression of C-KIT is consistent with previous reports of lack of response of pheochromocytomas to C-KIT inhibitor therapy. ${ }^{34}$

\section{FGFRs in Pheochromocytomas and Paragangliomas}

FGFR1, -2, and -4 are overexpressed in pheochromocytomas and paragangliomas; FGFR1 score and FGFR2 nuclear score were associated with an increased risk of metastasis. Even though there was no significant association between FGFR4 levels and outcomes, there was a positive correlation between FGFR4 levels and MIB1 LI in pheochromocytomas and with tumor size and weight in paragangliomas.

These findings suggest that FGFRs 1, 2, and 4 may be involved in pheochromocytoma/paraganglioma progression; this is supported by the observation that bFGF can act as a mitogen for rat chromaffin cells, especially in low oxygen concentrations or when associated with insulin-like growth factors, ${ }^{35,36}$ and can promote survival in rat pheochromocytoma PC12 cells. ${ }^{15}$ In addition, bFGF levels are higher in pheochromocytomas and paragangliomas than in normal adrenal medulla; ${ }^{3,15}$ both bFGF and FGFR1 are expressed in pheochromocytomas and at higher levels than in normal carotid body, pointing to a possible autocrine or paracrine mechanism for tumor development. ${ }^{16}$ We identified a higher FGFR1 score in SDH-related tumors; Dekker et al ${ }^{16}$ also showed higher staining intensity of FGFR1 and higher bFGF RNA levels in SDHD-mutated versus sporadic tumors.

A role for FGFRs in carcinogenesis and tumor progression has been well documented in various human malignancies, including prostate, colon, lung, breast and bladder cancer, multiple myeloma, and sarcomas. ${ }^{37}$ FGFR1 overexpression has been associated with liver metastasis in colorectal carcinoma. ${ }^{38}$

FGFR2 is more controversial as an oncogene, ${ }^{37}$ as in some models, such as pituitary ${ }^{10}$ and thyroid, ${ }^{8}$ it may act as a tumor suppressor. This might in part be due to alternative gene splicing that generates two common FGFR2 isoforms, which have different binding affinites to FGFs and are differentially expressed in normal tissues and tumors. Many tumors have shown a switch from the FGFR2 isoform expressed in their normal tissue of origin. $^{37}$ Moreover, the same FGFR2 isoform may have different properties that vary with the cell in which it is expressed (tumor epithelial versus stromal cells), and the interaction between these is what will determine the net effect of FGFR2 isoforms on a specific tumor growth and progression. ${ }^{8}$

Our finding that nuclear FGFR2 was associated with increased risk of metastasis and recurrence is not unprecedented. FGFRs can localize to the nucleus in normal and neoplastic cells, ${ }^{39}$ including those of the adrenal medulla. ${ }^{40,41}$ Nuclear FGFRs 

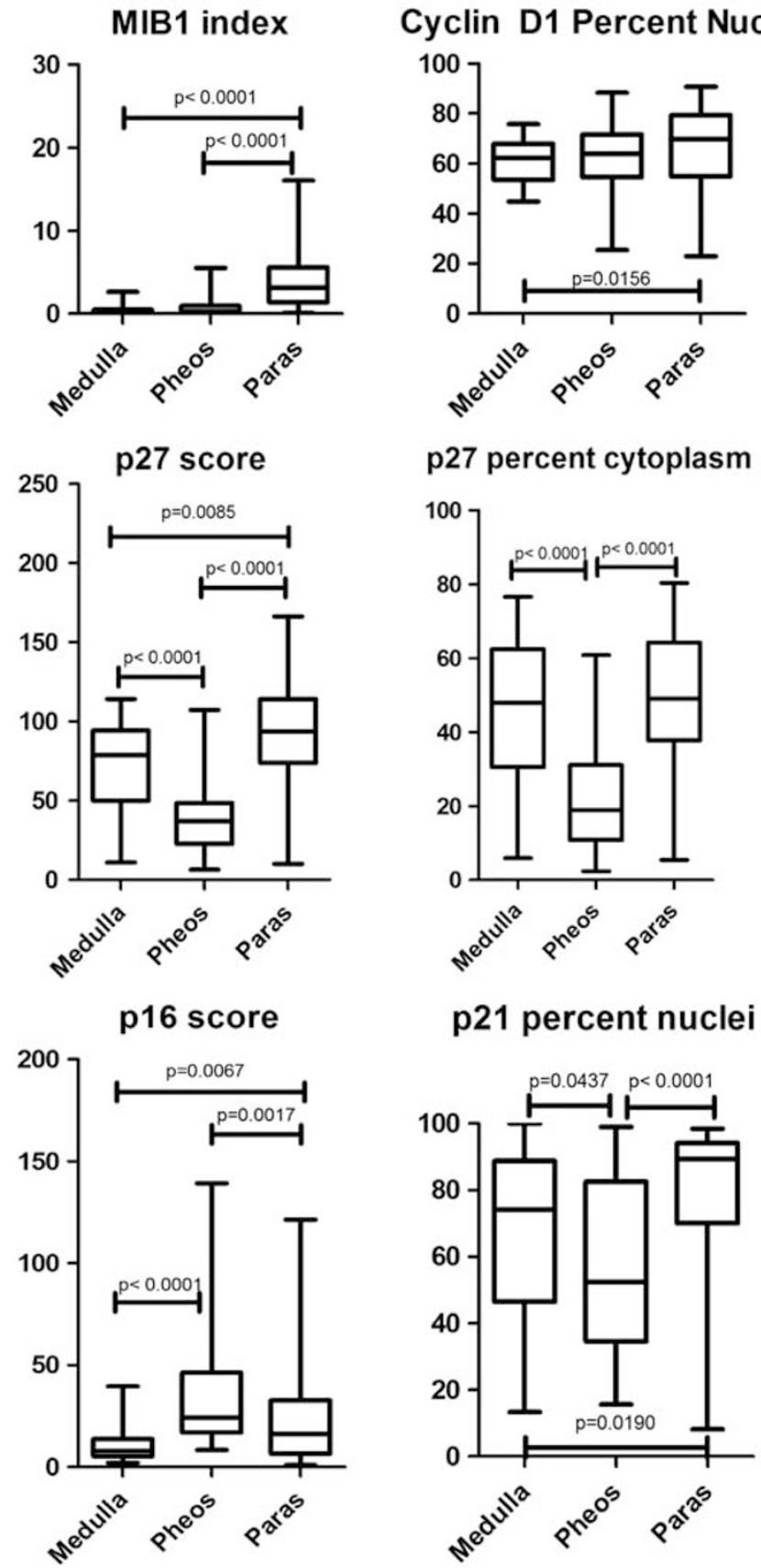

p27 percent cytoplasm

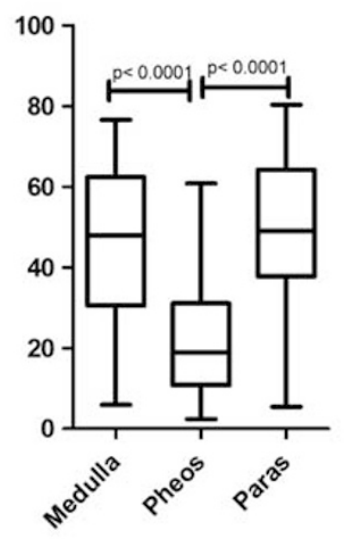

p21 percent nuclei

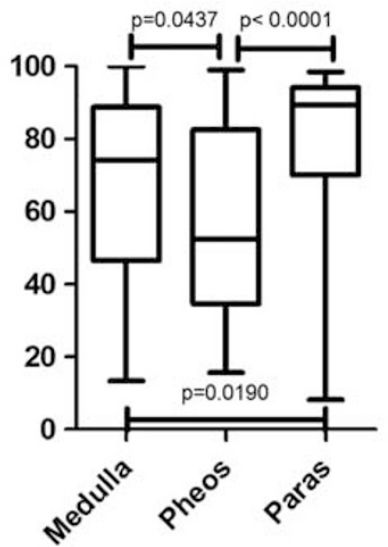

Rb percent nuclei

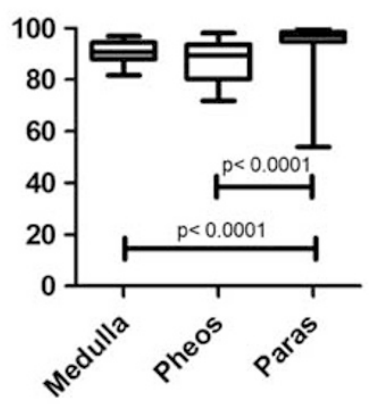

p27 percent nuclei

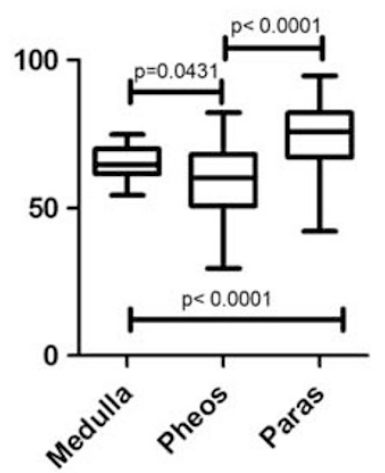

Aurora A Score

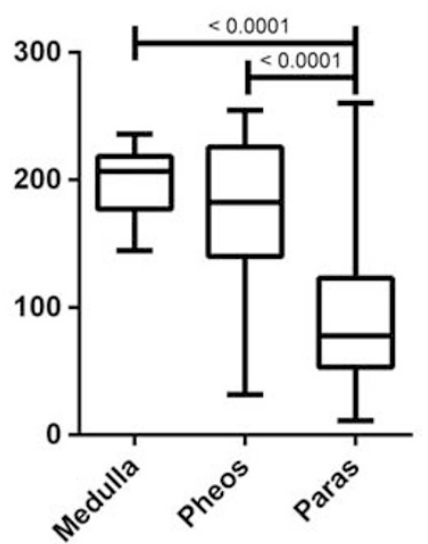

Figure 5 Differential expression of cell cycle markers in pheochromocytomas, paragangliomas, and normal adrenal medulla. Thirty-nine pheochromocytomas, 76 paragangliomas, and 35 normal human adult adrenomedullary tissue samples were compared. Unless otherwise specified, the heading 'score' refers to the overal staining score (values range: 0-300). MIB1 index refers to the percentage of nuclei staining for MIB1. Pheos, pheochromocytomas; paras, paragangliomas.

may be crucial in malignant transformation, as exemplified in a breast cancer model where FGFR1 activates genes involved in cell migration. ${ }^{39}$ Thus, both the isoform(s) expressed and the intracellular location of FGFRs seem to be important. However further investigation is required to determine the FGFR2 isoforms expressed in pheochromocytomas and paragangliomas.

Approximately $60 \%$ of patients in our series harbored the FGFR4-R388 SNP allele. Compared with normal population distributions (Table 2), there is a clear over-representation of this genotype in our patient population. Nevertheless, in contrast with other malignancies, ${ }^{17}$ there was no significant association between genotype and clinical characteristics or outcomes. This may be because of the relatively small sample size and of the low frequency of negative outcomes in our series. A higher FGFR4 score was found in familial and $S D H$ related tumors tumors. 
In contrast to FGFRs 1, 2, and 4, FGFR3 was underexpressed in paragangliomas, while overexpressed in pheochromocytomas. FGFR3 expression was significantly lower in tumors that metastasized, with high levels of FGFR3 being associated with a decreased risk of aggressive behavior. Membranous FGFR3 expression was inversely correlated with tumor size in paragangliomas. All these point to a tumor suppressive role for FGFR3 in pheochromocytomas and paragangliomas, which is supported by the association of FGFR3 overexpression/mutation with better prognosis in bladder ${ }^{42}$ and prostate carcinomas. ${ }^{43}$

\section{Cell Cycle Markers in Pheochromocytomas and Paragangliomas}

Given the well-known indolent growth of both pheochromocytomas and paragangliomas, overexpression of cell cycle inhibitors is not unexpected. Malignant tumors showed a higher MIB1 LI consistent with previous studies, ${ }^{3,4}$ and also a higher percentage of p27 cytoplasmic staining, which has not been previously reported in pheochromocytomas and paragangliomas.

Although p27 has been traditionally viewed as a tumor suppressor gene due to its ability to block cell cycle progression, there is increasing evidence that its role in tumorigenesis may go beyond cell cycle regulation. Cytoplasmic relocalization has been shown to be one of the mechanisms tumor cells develop to inactivate p27. In the cytoplasm, p27 not only cannot exert its inhibitory actions over cyclin CDKs, but may also actively participate in the process of malignant transformation. ${ }^{44}$ In keeping with that, cytoplasmic p27 has been associated with poor prognosis in carcinomas of the breast, cervix, esophagus, ovary, uterus, some leukemias and lymphomas, and in melanomas. ${ }^{44}$

The low percentage of p53 staining is in accordance with previous studies showing a very low frequency of p53 mutations in these tumors. ${ }^{45}$

Interestingly, although p21, p27, Cyclin D1, and $\mathrm{Rb}$ levels were higher in $S D H$-related tumors, p16 showed the reverse pattern. These findings are consistent with a previous report of p16ink4a promoter hypermethylation in $S D H B$-mutated and malignant tumors, ${ }^{46}$ and with the observation that pheochromocytoma-prone mice with homozygous Ink4a/Arf inactivation often develop malignant tumors. ${ }^{46}$

Aurora kinases are serine/threonine kinases that, together with cyclin-dependent kinases and Pololike kinases, are essential for proper progression of cell division through mitoses. ${ }^{47}$ Aurora A overexpression may contribute to genetic instability by disrupting the proper assembly of the mitotic checkpoint complex and has been detected in breast, colorectal, bladder, pancreatic, gastric, ovarian and esophageal cancers. ${ }^{47}$ No studies have thus far described the expression of Aurora kinases in pheochromocytomas and paragangliomas. We show that Aurora $\mathrm{A}$ is underexpressed in paragangliomas when compared with normal medulla, and that Aurora B expression is negligible in both tumors. This is consistent with the fact that most pheochromocytomas and paragangliomas have overall low levels of genetic instability ${ }^{48}$ and suggests that these tumors are not a suitable target for specific Aurora kinase inhibitor therapy.

Our findings provide novel insights into mechanistic actions of sunitinib in pheochromocytomas and paragangliomas, and give support to current evidence that multitargeted TKIs might be a suitable treatment alternative for pheochromocytomas and paragangliomas.

\section{Immunohistochemical Heat Maps and Hierarchical Clustering}

Hierarchical clustering based on the immunohistochemistry heat maps generated from our samples was consistent with previous observations that pheochromocytomas and paragangliomas, although sharing the same embryological origin, are a heterogeneous group of tumors that are better subclassified by their gene expression profiling. ${ }^{14,49}$ These studies have shown that pheochromocytomas and paragangliomas consistently seggregate into two clusters, one characterized by a pseudo-hypoxic gene signature and comprising $V H L$ - and $S D H$-related tumors, and the other including MEN- and NF1associated tumors, as well as most sporadic samples. ${ }^{14}$ In accordance with those previous studies, we have observed that $S D H$-related tumors show higher VEGFR1 and 2 levels when compared with MEN2 tumors, consistent with a so-called 'pseudo-hypoxic' gene signature in that cluster. The consistency of our findings with those previously reported in the literature not only helps to validate the use of automated immunohistochemistry analyses in our study but also the use of SDHB immunohistochemistry as an initial screening tool for identifying $S D H$-related tumors.

\section{Acknowledgments}

We thank Dr A Tischler for providing the MPC 4/30 cell line and Dr J Powers for his support and assistance in growing MPC cells. CAC was a recipient of the grant 'Strategic Training in Health Research (STIHR), STP-53912' at the time this research was conducted.

\section{Disclosure/conflict of interest}

The authors declare no conflict of interest. 


\section{References}

1 Lloyd RV, Tischler AS, Kimura N, et al. Adrenal tumors: introduction, In: DeLellis RA, Lloyd RV, Heitz PU, Eng C (eds). Pathology and Genetics of Tumours of Endocrine Organs. World Health Organization Classification of Tumours. IARC Press: Lyon, France; 2004, pp 137-138.

2 Gimenez-Roqueplo AP, Tischler AS. Pheochromocytoma and paraganglioma: progress on all fronts. Endocr Pathol 2012;23:1-3.

3 Clarke MR, Weyant RJ, Watson CG, et al. Prognostic markers in pheochromocytoma. Hum Pathol 1998;29:522-526.

4 August C, August K, Schroeder S, et al. CGH and CD 44/MIB-1 immunohistochemistry are helpful to distinguish metastasized from nonmetastasized sporadic pheochromocytomas. Mod Pathol 2004;17:1119-1128.

5 Ayala-Ramirez M, Feng L, Johnson MM, et al. Clinical risk factors for malignancy and overall survival in patients with pheochromocytomas and sympathetic paragangliomas: primary tumor size and primary tumor location as prognostic indicators. J Clin Endocrinol Metab 2011;96:717-725.

6 Joshua AM, Ezzat S, Asa SL, et al. Rationale and evidence for sunitinib in the treatment of malignant paraganglioma/pheochromocytoma. J Clin Endocrinol Metab 2009;94:5-9.

7 Ayala-Ramirez M, Chougnet CN, Habra MA, et al. Treatment with sunitinib for patients with progressive metastatic pheochromocytomas and sympathetic paragangliomas. J Clin Endocrinol Metab 2012;97: 4040-4050.

8 Guo M, Liu W, Serra S, et al. FGFR2 isoforms support epithelial-stromal interactions in thyroid cancer progression. Cancer Res 2012;72:2017-2027.

9 Kondo T, Zheng L, Liu W, et al. Epigenetically controlled fibroblast growth factor receptor 2 signalling imposes on the RAS/BRAF/mitogen-activated protein kinase pathway to modulate thyroid cancer progression. Cancer Res 2007;67:5461-5470.

10 Zhu X, Asa SL, Ezzat S. Fibroblast growth factor 2 and estrogen control the balance of histone 3 modifications targeting MAGE-A3 in pituitary neoplasia. Clin Cancer Res 2008;14:1984-1996.

11 Serra S, Zheng L, Hassan M, et al. The FGFR4-G388R single-nucleotide polymorphism alters pancreatic neuroendocrine tumor progression and response to mTOR inhibition therapy. Cancer Res 2012;72:5683-5691.

12 Takekoshi K, Isobe K, Yashiro T, et al. Expression of vascular endothelial growth factor (VEGF) and its cognate receptors in human pheochromocytomas. Life Sci 2004;74:863-871.

13 Salmenkivi K, Heikkilä P, Liu J, et al. VEGF in 105 pheochromocytomas: enhanced expression correlates with malignant outcome. APMIS 2003;111:458-464.

14 Favier J, Igaz P, Burnichon N, et al. Rationale for antiangiogenic therapy in pheochromocytoma and paraganglioma. Endocr Pathol 2012;23:34-42.

15 Statuto M, Ennas MG, Zamboni G, et al. Basic fibroblast growth factor in human pheochromocytoma: a biochemical and immunohistochemical study. Int J Cancer 1993;53:5-10.

16 Dekker PBD, Kuipers-Dijkshoorn NJ, Baelde HJ, et al. Basic fibroblast growth factor and fibroblastic growth factor receptor-1 may contribute to head and neck paraganglioma development by an autocrine or paracrine mechanism. Hum Pathol 2007;38:79-85.
$17 \mathrm{Xu} \mathrm{W,} \mathrm{Li} \mathrm{Y,} \mathrm{Wang} \mathrm{X,} \mathrm{et} \mathrm{al.} \mathrm{FGFR4} \mathrm{transmembrane}$ domain polymorphism and cancer risk: a meta-analysis including 8555 subjects. Eur J Cancer 2010;46: 3332-3338.

18 Ellis LM, Hicklin DJ. VEGF-targeted therapy: mechanisms of anti-tumour activity. Nat Rev Cancer 2008;8:579-591.

19 Saito Y, Tanaka Y, Aita Y, et al. Sunitinib induces apoptosis in pheochromocytoma tumor cells by inhibiting VEGFR2/Akt/mTOR/S6K1 pathways through modulation of Bcl-2 and BAD. Am J Physiol Endocrinol Metab 2012;302:E615-E625.

20 Powers JF, Evinger MJ, Tsokas P, et al. Pheochromocytoma cell lines from heterozygous neurofibromatosis knockout mice. Cell Tissue Res 2000;302: 309-320.

21 Kono SA, Marshall ME, Ware KE, et al. The fibroblast growth factor receptor signaling pathway as a mediator of intrinsic resistance to EGFR-specific tyrosine kinase inhibitors in non-small cell lung cancer. Drug Resist Update 2009;12:95-102.

22 van Nederveen FH, Gaal J, Favier J, et al. An immunohistochemical procedure to detect patients with paraganglioma and phaeochromocytoma with germline SDHB, SDHC, or SDHD gene mutations: a retrospective and prospective analysis. Lancet Oncol 2009;10:764-771.

23 Bange J, Prechtl D, Cheburkin Y, et al. Cancer progression and tumor cell motility are associated with the FGFR4 Arg(388) allele. Cancer Res 2002;62: 840-847.

24 Jensen LJ, Kuhn M, Stark M, et al. STRING 8 - a global view on proteins and their functional interactions in 630 organisms. Nucleic Acids Res 2009;37: D412-D416.

25 Saeed AI, Sharov V, White J, et al. TM4: a free, opensource system for microarray data management and analysis. Biotechniques 2003;34:374-378.

26 Karaca Z, Tanriverdi F, Unluhizarci K, et al. VEGFR1 expression is related to lymph node metastasis and serum VEGF may be a marker of progression in the follow-up of patients with differentiated thyroid carcinoma. Eur J Endocrinol 2011;164:277-284.

$27 \mathrm{Li} \mathrm{T}$, Zhu Y, Qin CY, et al. Expression and prognostic significance of vascular endothelial growth factor receptor 1 in hepatocellular carcinoma. J Clin Pathol 2012;65:808-814.

28 Heldin CH. Autocrine PDGF stimulation in malignancies. Ups J Med Sci 2012;117:83-91.

29 Zhang J, Wang P, Dykstra M, et al. Platelet-derived growth factor receptor- $\alpha$ promotes lymphatic metastases in papillary thyroid cancer. J Pathol 2012;228: 241-250.

30 Russell MR, Liu Q, Fatatis A. Targeting the alpha receptor for platelet-derived growth factor as a primary or combination therapy in a preclinical model of prostate cancer skeletal metastasis. Clin Cancer Res 2010;16:5002-5010.

31 Smith CL, Tallquist MD. PDGF function in diverse neural crest cell populations. Cell Adh Migr 2010;4: 561-566.

32 Schöllmann C, Grugel R, Tatje D, et al. Basic fibroblast growth factor modulates the mitogenic potency of the platelet-derived growth factor (PDGF) isoforms by specific upregulation of the PDGF alpha receptor in vascular smooth muscle cells. J Biol Chem 1992;267: 18032-18039. 
33 Frost EE, Nielsen JA, Le TQ, et al. PDGF and FGF2 regulate oligodendrocyte progenitor responses to demyelination. J Neurobiol 2003;54:457-472.

34 Koch CA, Gimm O, Vortmeyer AO, et al. Does the expression of c-kit (CD117) in neuroendocrine tumors represent a target for therapy? Ann N Y Acad Sci 2006;1073:517-526.

35 Nurse CA, Vollmer C. Role of basic FGF and oxygen in control of proliferation, survival, and neuronal differentiation in carotid body chromaffin cells. Dev Biol 1997;184:197-206.

36 Frödin M, Gammeltoft S. Insulin-like growth factors act synergistically with basic fibroblast growth factor and nerve growth factor to promote chromaffin cell proliferation. Proc Natl Acad Sci USA 1994;91: 1771-1775.

37 Brooks AN, Kilgour E, Smith PD. Molecular pathways: fibroblast growth factor signaling: a new therapeutic opportunity in cancer. Clin Cancer Res 2012;18: 1855-1862.

38 Sato T, Oshima T, Yoshihara K, et al. Overexpression of the fibroblast growth factor receptor-1 gene correlates with liver metastasis in colorectal cancer. Oncol Rep 2009;21:211-216.

39 Chioni AM, Grose R. FGFR1 cleavage and nuclear translocation regulates breast cancer cell behavior. J Cell Biol 2012;197:801-817.

40 Stachowiak MK, Maher PA, Joy A, Gaal J, et al. Nuclear accumulation of fibroblast growth factor receptors is regulated by multiple signals in adrenal medullary cells. Mol Biol Cell 1996;7:1299-1317.

41 Peng H, Moffett J, Myers J, et al. Novel nuclear signalling pathway mediates activation of fibroblast growth factor-2 gene by type 1 and type 2 angiotensin II receptors. Mol Biol Cell 2001;12:449-462.

42 van Rhijn BW, van der Kwast TH, Liu L, et al. The FGFR3 mutation is related to favorable pT1 bladder cancer. J Urol 2012;187:310-314.

43 Hernandez S, de MS, Agell L, et al. FGFR3 mutations in prostate cancer: association with low-grade tumors. Mod Pathol 2009;22:848-856.

44 Serres MP, Zlotek-Zlotkiewicz E, Concha C, et al. Cytoplasmic p27 is oncogenic and cooperates with Ras both in vivo and in vitro. Oncogene 2011;30: 2846-2858.

45 Petri BJ, Speel EJ, Korpershoek E, et al. Frequent loss of 17p, but no p53 mutations or protein overexpression in benign and malignant pheochromocytomas. Mod Pathol 2008;21:407-413.

46 Kiss NB, Geli J, Lundberg F, et al. Methylation of the p16INK4A promoter is associated with malignant behavior in abdominal extra-adrenal paragangliomas but not pheochromocytomas. Endocr Relat Cancer 2008;15:609-621.

47 Saeki T, Ouchi M, Ouchi T. Physiological and oncogenic Aurora-A pathway. Int J Biol Sci 2009;5: 758-762.

48 Sandgren J, Diaz de Ståhl T, Andersson R, et al. Recurrent genomic alterations in benign and malignant pheochromocytomas and paragangliomas revealed by whole-genome array comparative genomic hybridization analysis. Endocr Relat Cancer 2010;17:561-579.

49 Dahia PL, Ross KN, Wright Me, et al. A HIF1 alpha regulatory loop links hypoxia and mitochondrial signals in pheochromocytomas. PLoS Genet 2005;1: 72-80.

Supplementary Information accompanies the paper on Modern Pathology website (http://www.nature.com/ modpathol) 